\title{
Attentional capture and inhibition (of return): The effect on perceptual sensitivity
}

\author{
JAN THEEUWES and CHI YONG DONNY CHEN \\ Vrije Universiteit Amsterdam, Amsterdam, The Netherlands
}

\begin{abstract}
The present study was designed to determine the spatial distribution of attention in displays in which an irrelevant color singleton was present. The results show that at the location of an irrelevant singleton, there is, first, an increased sensitivity $\left(d^{\prime}\right)$, followed later by a reduced sensitivity. The increased sensitivity implies that, first, the irrelevant singleton captured spatial attention, producing an increased sensory gain for input at the irrelevant distractor location. The later-occurring, reduced sensitivity implies the operation of inhibitory processes, possibly related to inhibition of return.
\end{abstract}

One of the most basic questions in the study of attention is the extent to which top-down attentional control can prevent distraction by irrelevant stimuli. There are two ways in which selection may be controlled. On the one hand, attentional control is thought to be goal-directed when attentional priority is given to only those objects and events that are in line with the current goals of the observer. On the other hand, selection is thought to occur in a stimulus-driven manner when, irrespective of the intentions or goals of the observer, objects and events involuntarily receive attentional priority, a phenomenon referred to as attentional capture (for recent reviews, see, e.g., Ruz \& Lupiáñez, 2002; Theeuwes \& Godijn, 2001; Yantis \& Egeth, 1999).

In the early $1990 \mathrm{~s}$, Theeuwes $(1991,1992,1994)$ proposed that in early vision, attentional selection is exclusively stimulus driven, with the possibility of top-down control only later in processing (Theeuwes, 2004; Theeuwes, Atchley, \& Kramer, 2000). Theeuwes used a visual search task that has become known as the additional singleton task, in which one item in the display is the target singleton, whereas another singleton is completely unrelated and irrelevant to the task. This condition is compared with a condition in which the irrelevant singleton is not present. For example, Theeuwes (1992) presented participants with circular displays consisting of identically colored circles and one diamond. Line segments of different orientations appeared in the circles and diamonds, and the participants had to determine the orientation of the line segment appearing in the target shape. The target shape that the participants searched for was a singleton, because it was the only diamond present in the display. In the distractor condition, an irrelevant color singleton

We thank Charles L. Folk, Juan Lupiáñez, and Richard Abrams for their excellent comments and suggestions on an earlier version of the manuscript. Correspondence concerning this article should be addressed to J. Theeuwes, Department of Cognitive Psychology, Vrije Universiteit, Van der Boechorststraat 1, 1081 BT Amsterdam, The Netherlands (e-mail: j.theeuwes@psy.vu.nl). was also present in the display. Time to find the shape singleton increased when an irrelevant color singleton was present (i.e., one of the circles was red). Theeuwes (1991, 1992, 1994, 1996, 2004) explained the increase in search time in conditions in which an irrelevant singleton was present in terms of attentional capture. Because attention was automatically captured by the distractor singleton (the most salient element in the display), it took longer before attention could be redirected to the location of the target singleton and a response could be emitted. In a recent study, Theeuwes, Kramer, and Kingstone (2004) demonstrated that presence of an irrelevant singleton also modulated target detectability $\left(d^{\prime}\right)$. In line with earlier claims, Theeuwes et al. (2004) argued that the capture of attention by the irrelevant singleton causes a reduced sensory input at the target location. Irrespective of the attentional set of the observer, spatial attention was first captured by the salient distractor singleton before it could be redirected to the target singleton, and this caused a reduced $d^{\prime}$ at the location of the target.

The notion suggested by Theeuwes is similar to that of Koch and Ullman (1985), who introduced the notion of a saliency map to accomplish preattentive selection. This map is a two-dimensional topographical map that encodes the saliency of objects in their visual environment. Neurons in this map compete among each other, giving rise to a single winning location (i.e., winner takes all) that contains the most salient element. The saliency map is the result of a preattentive parallel encoding across the visual field in which differences in simple visual features, such as intensity, contrast, color, and orientation, are calculated. Focused spatial attention simply scans the locations of decreasing activation (saliency). If a location is inhibited, the next salient location will receive spatial attention (see also Itti \& Koch, 2001; Sagi \& Julesz, 1984).

Contrary to the notion that attention is driven solely by bottom-up factors, others have claimed that the ability of a singleton to capture attention is contingent on whether an attentional-capturing stimulus is consistent with topdown settings, which are established offline on the basis 
of current attentional goals (Folk \& Remington, 1998; Folk, Remington, \& Johnston, 1992). According to the contingent capture model, only stimuli that match the topdown control settings will capture attention; stimuli that do not match the top-down settings will be ignored. Thus, according to this theory, top-down control is possible even when both the target and the distractor are singletons. Along these lines, it was argued that in Theeuwes's experiments, the irrelevant singleton captured attention because the participants were set to find a singleton (e.g., a local mismatch), rather than a particular feature, such as a red circle (see Bacon \& Egeth's, 1994, notion of feature search and singleton detection modes; but see Theeuwes, 2004). It is claimed that irrespective of bottom-up saliency, the singleton that matches the top-down setting will capture attention. The evidence for these claims is based on experiments in which participants had to ignore a cue that appeared $150 \mathrm{msec}$ prior to the presentation of the target display (Folk et al., 1992). The participants responded to a character shape (X vs. $=$ ) that, in different conditions, had either a unique color or a unique abrupt onset. When the search display was preceded by a tobe-ignored feature singleton (the cue) that matched the singleton for which they were searching, the cue captured attention, as evidenced by a prolonged reaction time (RT) to identify the target (i.e., when the cue and the target appeared in different spatial locations). On the other hand, if the to-be-ignored feature singleton (the cue) did not match the singleton for which they were searching, its appearance apparently did not capture attention. This contingent capture of attention occurred for both color and onset conditions and is considered evidence that involuntary capture is contingent on the adoption of an attentional set. The critical finding in these studies is that a cue that does not match the top-down search goal (i.e., the defining property of the target) does not affect RT, whereas a cue that matches the search goal has an effect on RT. In other words, if participants were searching for a red plus sign, they were more likely to be distracted by a red cue than by a cue that was an abrupt onset, and vice versa. Folk et al. suggested that the absence of an effect on RT for a cue that does not match the target indicates that the cue did not capture attention.

To explain the discrepancy between the results of Folk et al. (1992) and Theeuwes $(1992,1994)$, Theeuwes et al. (2000) took the original displays of Theeuwes (1992) but presented the irrelevant color singleton at various stimulus onset asynchronies (SOAs) prior to the presentation of the target displays. Relative to the no-distractor condition, the additional singleton produced significant costs at the short SOAs (i.e., 50 and $100 \mathrm{msec}$ ) but had no effect on RT at an SOA greater than $100 \mathrm{msec}$. These findings indicated that attentional capture always occurs, yet the system is able to recover within $150 \mathrm{msec}$. Thus, these findings suggest that the results obtained with the spatial cuing paradigm of Folk and colleagues (Folk \& Remington, 1998; Folk et al., 1992), in which the cue to be ignored is presented
$150 \mathrm{msec}$ before the search display, may not be conclusive regarding the presence or absence of capture, because within $150 \mathrm{msec}$ it is possible to obtain efficient recovery from capture (for similar findings, see also Kim \& Cave, 1999; Lamy \& Egeth, 2003; Lamy, Tsal, \& Egeth, 2003).

Even though there appears to be strong evidence for bottom-up capture by irrelevant singletons, followed by rapid disengagement and recovery, one may argue that Theeuwes's experiments never showed direct evidence for the existence of capture, because there was never an explicit manipulation of the spatial relationship between the target and the distractor (see Folk \& Remington, in press). Indeed, in all of Theeuwes's experiments (1990, 1991, 1992, 1994, 1996; Theeuwes et al., 2000; Theeuwes \& Godijn, 2002), the target never appeared at the same location as the distractor. The occurrence of capture had to be inferred on the basis of an increase in RT relative to the no-distractor condition (Theeuwes, 1991, 1992), the presence of a compatibility effect between the target and the distractor (Theeuwes, 1996), the occurrence of inhibition of return (IOR) at the location of the distractor (Theeuwes \& Godijn, 2002), or a decrease in $d^{\prime}$ when a distractor was present (Theeuwes et al., 2004).

Since there was never a systematic manipulation of target and distractor locations in Theeuwes's experiments, one still could argue that there was no capture of attention to the location of the most salient singleton. For example, Folk and Remington (1998) argued that an increase in RT in the additional singleton paradigm in conditions in which a distractor was present was not due to attentional capture but, instead, to so-called filtering costs, a notion introduced by Kahneman, Treisman, and Burkell (1983). The idea of filtering costs is that the presence of an irrelevant singleton may slow the deployment of attention to the target item by requiring an effortful and time-consuming filtering operation. According to this line of reasoning, attention is employed in a top-down way and goes directly to the target singleton; simply because another irrelevant singleton is present, directing attention to the target may take more time than when no irrelevant singleton is present. Note that this view does not entail a shift of spatial attention to the location of the irrelevant singleton.

In order to determine whether irrelevant singletons capture spatial attention in the present study, we explicitly manipulated the relationship between target and distractor locations (see also, e.g., Turatto \& Galfano, 2001, for a similar manipulation). Similar to the spatial-cuing task in Folk et al. (1992), the irrelevant distractor singleton could appear at a location at which the target would appear somewhat later.

To determine capture, we used the same method as that in a previous study (Theeuwes et al., 2004; see also Handy, Jha, \& Mangun, 1999; Handy, Kingstone, \& Mangun, 1996), which is basically derived from signal detection theory. Unlike RTs, signal detection measures allow for the separation of perceptual and decision-level effects of attention. Participants were required to make a forced 
two-choice decision regarding the orientation of a small target bar, which was consistently located in the shape singleton (a gray diamond among gray circles).

\section{EXPERIMENT 1}

The participants searched for a gray diamond presented among five gray circles (similar to search in Theeuwes et al., 2000). The search display was preceded by a distractor display containing an irrelevant red distractor among five gray nontarget elements (see Figure 1). The distractor singleton was equally likely to appear at any of the six possible display locations. Therefore, in one sixth of the trials, the distractor singleton was presented at the location at which the target would appear $60 \mathrm{msec}$ later. Note that we used a 60 -msec SOA between the presentations of the distractor and the target singleton to ensure that, in case the distractor singleton did capture spatial attention, attention would be at that location when the target arrived. Indeed, as was argued in Theeuwes et al. (2000), when the interval is longer than $100 \mathrm{msec}$ - as for example, was the case in Folk et al.'s (1992) paradigm - one may not find any effects of attentional allocation at the distractor singleton location, because a long interval allows efficient recovery from capture.

In distractor trials, the irrelevant red color singleton was presented at chance level at the location at which the target singleton would appear $60 \mathrm{msec}$ later. The question was whether the $d^{\prime}$ for this location would be higher than that for any of the other locations. An increase in $d^{\prime}$ at this location would provide clear evidence for attentional capture.

\section{Method}

Participants. Eight participants with normal or corrected-tonormal vision took part in the experiment. All had self-reported normal or corrected-to-normal vision and reported having no color vision defects.

Stimuli. The display was similar to that in Theeuwes et al. (2000). Six gray display elements were equally spaced around the fixation point on an imaginary circle ( $6.6^{\circ}$ radius). Each element consisted of the outline of a circle $\left(2.4^{\circ}\right.$ in diameter $)$ and a superimposed outline of a diamond $\left(2.4^{\circ}\right.$ on a side). All the elements were equiluminant at $5.0 \mathrm{~cd} / \mathrm{m}^{2}$. The distractor display was basically the same as the begin display, with the exception that one of the six elements was colored red (distractor singleton; $5.0 \mathrm{~cd} / \mathrm{m}^{2}$ ). The Euclidian distances, center to center, between the target and the distractor singleton were $6.7^{\circ}$, $11.5^{\circ}$, or $13.2^{\circ}$. The distance between the target and the distractor singleton was systematically varied within blocks. The search display appeared by removing five diamonds and one circle from the distractor display. Within each outline circle in the search display, there was one diagonal line segment $\left(0.8^{\circ}\right.$ in length). The target line segment (initially set at a length of $1.0^{\circ}$ ) was always located within the outline of the diamond (target singleton) and had either a vertical or a horizontal orientation. Note that a vertical or horizontal line segment does not pop out among slightly tilted line segments (see Theeuwes, 1991), which makes it highly unlikely that the partici-

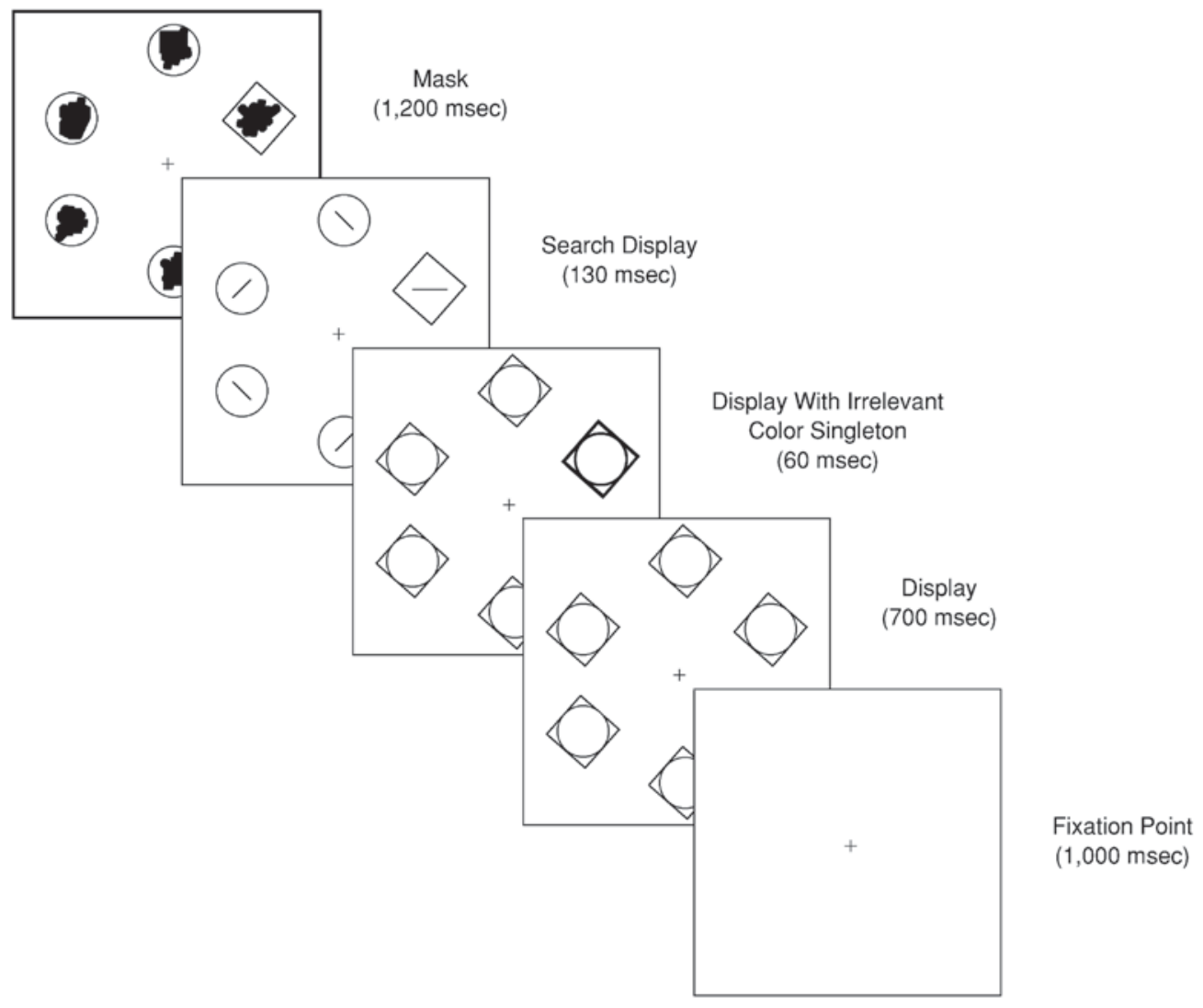

Figure 1.A graphical illustration of the sequence and timing of stimulus events presented on each trial. 
pants searched directly for the target line segment, instead of for the shape singleton (the diamond) that contained the target line segment. The mask consisted of randomly filled gray line segments, which would appear over the line segments. All the line segments and the masks were equiluminant at $6.0 \mathrm{~cd} / \mathrm{m}^{2}$. Figure 1 gives an example of a trial. In the example, the colored singleton happened to be presented at the same location as the target singleton.

Procedure. After the fixation point had been presented for $1,000 \mathrm{msec}$, the initial display was presented for $700 \mathrm{msec}$. If a color distractor singleton was present, one of the elements of the initial display turned red for $60 \mathrm{msec}$. The search display, which was created by removing five diamonds and one circle, was presented for $130 \mathrm{msec}$, followed by a mask. The target line segment, either horizontal or vertical, was always located in the diamond. The line segments in the other five circles were diagonals. The participants were required to direct their attention to the shape singleton and to make a discrimination judgment of the target bar's orientation (i.e., vertical or horizontal) inside the shape singleton. They were instructed to keep their eyes fixated at the fixation point. The participants were required only to respond (i.e., go trials) to the designated orientation (the target orientation, which was counterbalanced across participants). A hit was defined as a response on trials in which the designated target orientation was present, and a false alarm was defined as a response on trials in which the designated target orientation was absent. Accuracy was stressed. When the participants committed an error (miss or false alarm), a tone sounded. The participants were instructed to remain fixated on the central fixation point during the course of a trial. The total exposure duration of the display with the irrelevant singleton and the search display was $190 \mathrm{msec}$, a time frame that is considered to be too short to make effective eye movements.

In the present experiment, we used exactly the same adjustment procedure as that in Theeuwes et al. (2004). In order to minimize the possibility of floor or ceiling effects in accuracy, target line length was adjusted online to ensure that performance remained near $75 \%$ correct. Every 10 trials, the overall performance (hits and false alarms) was calculated. If overall accuracy dropped below $65 \%$, line length was increased by $0.1^{\circ}$. If performance was better than $85 \%$, line length was reduced by $0.1^{\circ}$. The line length procedure was carried out every 10 trials. Because no-distractor and distractor conditions were randomized in blocks of trials, the line adjustment procedure was based on both no-distractor and distractor trials. This ensured that overall, the same adjustment procedure was used for both conditions.

Design. The experiment consisted of 1,008 experimental trials, which were divided into four sessions of 252 trials. The practice session also consisted of 252 trials. The participants received feedback about their false alarms and misses after every 63 trials. When a colored distractor singleton was present, it appeared at the location of the target at chance level - that is, on one sixth of the trials. On fivesixths of the trials, the distractor singleton appeared equally often at any of the other five nontarget locations. Of the 1,008 experimental trials, 864 were distractor trials, and 144 were no-distractor trials in which no colored singleton was presented.

\section{Results}

The $d^{\prime}$ and $A^{\prime}$ measures were calculated for each participant. ${ }^{1}$ Table 1 presents the mean $d^{\prime}$ s, hit rates, false alarm rates, and $A^{\prime}$ s for the no-distractor and distractor conditions. An ANOVA on $d^{\prime}$ showed a main effect of condition $\left[F(4,28)=9.9, M S_{\mathrm{e}}=0.20, p<.01\right]$. Additional planned comparisons with distance as a factor showed that when the distractor singleton preceded the target singleton, the $d^{\prime}$ at that location was significantly higher than that at any other location (all $p \mathrm{~s}<.05$ ). Thus, $d^{\prime}$ at the location that had previously contained a distractor singleton was higher than it was when no distractor had been present, and it was higher than it was when the distractor singleton was present but not located at the target location but at some distance away from the target location (Locations 1, 2, and $3)$. There was no effect of distance on $d^{\prime}$.

\section{Discussion}

The results are clear. There was a large increase in $d^{\prime}$ for target detection at the location at which the target was preceded by a distractor singleton. In line with earlier cuing studies that showed that directing spatial attention to a location in space can enhance gains for inputs (e.g., Handy et al., 1999; Hawkins et al., 1990), the present findings show that attentional capture by an irrelevant color singleton also can enhance gains for inputs. Indeed, target detectability was higher at the location of the distractor singleton, providing unequivocal evidence for a shift of spatial attention to the location of the distractor singleton. Even though previous results of the additional singleton paradigm may have been explained in terms of attentional filtering (Folk \& Remington, 1998), the present findings show increased sensitivity for detecting targets that appear at a distractor singleton location. The present findings provide convincing evidence that attention was shifted to the location of the distractor singleton.

\section{EXPERIMENT 2}

The increased sensitivity at the location of the irrelevant distractor singleton confirms and elaborates the results of previous RT studies by Theeuwes (1991, 1992, 1994, 2004) that showed that spatial attention was captured exogenously by the irrelevant singleton. However, in line with the notion offered by Bacon and Egeth (1994), one may argue that the irrelevant singleton did not capture attention exogenously but that the participants chose in a top-down

Table 1

Mean $d^{\prime}$, Mean $A^{\prime}$, Hit Rate, and False Alarm Rate for the Distractor Singleton, the Target-Distractor Distance, and the No-Distractor Baseline (With Standard Deviations) in Experiment 1

\begin{tabular}{|c|c|c|c|c|c|c|c|c|}
\hline & \multicolumn{2}{|c|}{ Mean $d^{\prime}$} & \multicolumn{2}{|c|}{ Mean $A^{\prime}$} & \multicolumn{2}{|c|}{ Hit Rate } & \multicolumn{2}{|c|}{ False Alarm Rate } \\
\hline & $M$ & $S D$ & $M$ & $S D$ & $M$ & $S D$ & $M$ & $S D$ \\
\hline Distractor singleton & 2.626 & 0.276 & 0.932 & 0.019 & 0.859 & 0.043 & 0.092 & 0.020 \\
\hline Distance $=1$ & 1.391 & 0.100 & 0.830 & 0.013 & 0.681 & 0.031 & 0.198 & 0.033 \\
\hline Distance $=2$ & 1.505 & 0.098 & 0.843 & 0.011 & 0.708 & 0.031 & 0.197 & 0.041 \\
\hline Distance $=3$ & 1.629 & 0.125 & 0.859 & 0.010 & 0.741 & 0.033 & 0.191 & 0.035 \\
\hline No distractor & 1.556 & 0.132 & 0.850 & 0.016 & 0.736 & 0.022 & 0.201 & 0.044 \\
\hline
\end{tabular}


fashion to search for singletons (the singleton detection mode), instead of searching for a particular feature (the feature search mode). Experiment 2 addressed this issue by making search using a singleton detection mode impossible. In line with Bacon and Egeth (Experiment 3), the singleton detection strategy was eliminated by adding another unique shape. Rather than all the nontargets having the same shape (all circles) in this experiment, one of the circles was replaced by a square. Thus, on each trial, there were four nontarget circles and two unique shapes: one diamond, which was the target, and one square, which was a nontarget. According to Bacon and Egeth, the singleton detection strategy would not work anymore because, in this experiment, the target shape was not the only item that was unique with respect to form. In line with Bacon and Egeth, one would expect that the participants would have to use a feature search mode that should allow full top-down control. Therefore, one would expect that attention would not be captured by the irrelevant color singleton anymore and that there would be no increase in $d^{\prime}$ at the color singleton distractor location. If, however, search modes (i.e., if they exist; see Theeuwes, 2004) had nothing to do with the present findings, we would expect basically the same results as those in Experiment 1.

\section{Method}

Participants. Eight participants (5 men and 3 women) took part in this experiment.

Stimuli, Design, and Procedure. The experiment was identical to Experiment 1, except that one of the gray nontarget circles was replaced by a gray square. Note that squares were present at all locations in the premask display. By switching of squares, circles, or diamonds, we created a display that consisted of four circles, one square, and one diamond (the target).

\section{Results}

Table 2 presents the mean $d^{\prime}$ s, hit rates, false alarm rates, and $A^{\prime}$ 's for the no-distractor and distractor conditions. An ANOVA on $d^{\prime}$ showed a main effect of condition $\left[F(4,28)=4.52, M S_{\mathrm{e}}=0.066, p<.01\right]$. Additional planned comparisons showed that when the distractor singleton preceded the target singleton, the $d^{\prime}$ at that location was significantly higher than that in any of the other conditions (all $p \mathrm{~s}<.05$ ).

\section{Discussion}

The present results are clear: Even though the participants could not rely on a singleton detection strategy, there was still an increased sensitivity at the location of the distractor singleton. The results of Experiment 2 were basically the same as those of Experiment 1 . The present experiment adds to the growing literature suggesting that the distinction between a singleton detection and a feature search mode may not be as clear-cut as previously assumed (see also Theeuwes, 2004).

It should be noted that in both Experiments 1 and 2, the presence of a color distractor singleton at nontarget locations did not produce a decrement in sensitivity at the target location. Indeed, the $d^{\prime}$ when a distractor was present at a nontarget location was not different from that at the no-distractor location. This result is inconsistent with Theeuwes's $(1991,1992,1994)$ earlier findings, which showed that RT to a target singleton increases when a color singleton is present. More important, it is also inconsistent with recent data using $d^{\prime}$ as the main dependent measure (Theeuwes et al., 2004). In this study, it was shown that the presence of a color singleton at a nontarget location produced a decrement in sensitivity at the target location. The most likely reason for this discrepancy in results is that in the present study, the color distractor singleton was presented (and given the procedure, could only be presented) for a very short time interval $(60 \mathrm{msec})$ and then removed. In all of Theeuwes's previous studies, the color distractor singleton remained on the screen simultaneously with the target singleton until a response was given. In the present study, immediately following the presentation of the color distractor singleton, the search display was presented. Therefore, unlike in previous experiments, in the present study, the search display never contained a color distractor singleton. The absence of a color distractor singleton may have allowed a fast and efficient disengagement of attention from the location that earlier contained the color singleton. Because of this fast disengagement, there were virtually no costs for displays that earlier had contained a color singleton, relative to displays that did not contain such a singleton. Note that such fast disengagement will not occur when the location to which attention is captured happens to contain the target

Table 2

Mean $d^{\prime}$, Mean $A^{\prime}$, Hit Rate, and False Alarm Rate for the Distractor Singleton, the Target-Distractor Distance, and the No-Distractor Baseline (With Standard Deviations) in Experiment 2

\begin{tabular}{|c|c|c|c|c|c|c|c|c|}
\hline & \multicolumn{2}{|c|}{ Mean $d^{\prime}$} & \multicolumn{2}{|c|}{ Mean $A^{\prime}$} & \multicolumn{2}{|c|}{ Hit Rate } & \multicolumn{2}{|c|}{ False Alarm Rate } \\
\hline & $M$ & $S D$ & $M$ & $S D$ & $M$ & $S D$ & $M$ & $S D$ \\
\hline Distractor singleton & 1.644 & 0.178 & 0.859 & 0.021 & 0.762 & 0.041 & 0.194 & 0.023 \\
\hline Dist & 1.361 & 0.15 & 0.789 & & 0.656 & 0.029 & .229 & 0.034 \\
\hline Distance $=2$ & 1.393 & 0.108 & 0.812 & 0.015 & 0.648 & 0.035 & 0.209 & 0.02 \\
\hline Distance $=3$ & 1.140 & 0.197 & 0.784 & 0.030 & 0.632 & 0.062 & 0.234 & 0.04 \\
\hline No distractor & 1.223 & 0.112 & 0.808 & 0.018 & 0.696 & 0.031 & 0.252 & 0.035 \\
\hline
\end{tabular}


line segment. In this condition, attention remains at this location, explaining the high sensitivity $\left(d^{\prime}\right)$ for detecting the target line segment.

\section{EXPERIMENT 3}

Theeuwes et al. (2000) showed that once attention is exogenously captured by an irrelevant singleton, it takes only a very brief time to disengage attention from that location. In Theeuwes et al. (2000), prior to the presentation of the target display at different SOAs $(50,100,150,200$, 250 , and $300 \mathrm{msec}$ ), a color singleton was presented. The results showed that the presence of an irrelevant distractor singleton had an effect only when the target singleton and the distractor were presented in close succession (at 50and 100-msec SOAs). Theeuwes et al. (2000) argued that in conditions in which the target and the distractor were presented in close temporal proximity, there was not enough time to exert top-down control that could have overcome attentional capture by the salient distractor. However, when the distractor singleton was presented a considerable time before the presentation of the target singleton, it was possible to exert sufficient top-down control, such that by the time the target singleton was presented, there was no sign of attentional capture by the distractor anymore. In order to account for unexpected compatibility effects in Experiments 2 and 3, Theeuwes et al. (2000) suggested that "to gain attentional control, subjects may have inhibited the distractor location" (p. 116). Theeuwes et al.'s (2000) main findings were recently confirmed by Lamy and Egeth (2003), who showed that capture was indeed short lived (50- to $100-\mathrm{msec}$ SOA). More important, there was some evidence for Theeuwes et al.'s (2000) suggestion that spatial inhibition may have allowed a fast disengagement of attention from the distractor location. In Lamy and Egeth's Experiment 1, there was a nonsignificant trend showing search times that were higher when a target singleton appeared at a location that had earlier contained a distractor singleton. This increase in RT for targets appearing at locations that previously had contained a distractor singleton indeed suggests such an inhibitory mechanism. Lamy and Egeth argued that attentional deallocation is mediated by spatial inhibition, allowing for recovery from capture. ${ }^{2}$

In Experiment 3, we wanted to determine whether attentional deallocation is, indeed, mediated by spatial inhibition, as was suggested by Lamy and Egeth (2003) and
Theeuwes et al. (2000). We used the same paradigm as that in Experiment 1, except that we inserted an interval of $500 \mathrm{msec}$ in which no distractor was present. If the location of the distractor singleton is inhibited, we would expect a decrease in $d^{\prime}$ for the location that contained a distractor singleton.

\section{Method}

Participants. Twelve participants ( 8 men and 4 women) took part in this experiment.

Stimuli, Design, and Procedure. The experiment was identical to Experiment 1, except that we used a 150-msec display that contained a color distractor singleton. This display was followed by a no-distractor display (a display identical to the initial display; see Figure 1), which was presented for $500 \mathrm{msec}$. As in Experiment 1, the search display was revealed for $130 \mathrm{msec}$, followed by a mask. Again, it was stressed that the participants should keep their eyes fixated in the center.

\section{Results}

Table 3 presents the mean $d^{\prime}$ s, hit rates, false alarm rates, and $A^{\prime}$ s for the no-distractor and distractor conditions. An ANOVA on $d^{\prime}$ showed a main effect of condition $\left[F(4,44)=3.55, M S_{\mathrm{e}}=0.064, p<.05\right]$. Additional planned comparisons showed that when the distractor singleton preceded the target singleton, the $d^{\prime}$ at that location was significantly lower than that in any of the other conditions (all $p \mathrm{~s}<.05$ ). Thus, $d^{\prime}$ at the location that previously had contained a distractor singleton was lower than it was when no distractor had been present, and it was lower than it was when the distractor singleton was present but was not located at the target location but at some distance away from the target location (Locations 1, 2, and 3). There was no effect of distance on $d^{\prime}$.

\section{Discussion}

Whereas Experiments 1 and 2 showed an increased $d^{\prime}$ at the location of the distractor singleton, Experiment 3, which had a much longer interval between the distractor and the target singleton, showed a reduced $d^{\prime}$. These results suggest that attentional capture, as evidenced by a higher sensitivity, is followed by inhibition, as evidenced by a reduced sensitivity. In line with suggestions of Lamy and Egeth (2003) and Theeuwes et al. (2000), these findings seem to suggest that attentional capture is followed by inhibition. Inhibition may be necessary to allow a disengagement of attention at the location of the distractor singleton.

Table 3

Mean $d^{\prime}$, Mean $A^{\prime}$, Hit Rate, and False Alarm Rate for the Distractor Singleton, the Target-Distractor Distance, and the No-Distractor Baseline (With Standard Deviations) in Experiment 3

\begin{tabular}{|c|c|c|c|c|c|c|c|c|}
\hline & \multicolumn{2}{|c|}{ Mean $d^{\prime}$} & \multicolumn{2}{|c|}{ Mean $A^{\prime}$} & \multicolumn{2}{|c|}{ Hit Rate } & \multicolumn{2}{|c|}{ False Alarm Rate } \\
\hline & $M$ & $S D$ & $M$ & $S D$ & $M$ & $S D$ & $M$ & $S D$ \\
\hline Distractor singleton & 1.431 & 0.097 & 0.837 & 0.012 & 0.715 & 0.026 & 0.208 & 0.022 \\
\hline Distan & 1.698 & 0.064 & 0.872 & 0.007 & 0.777 & 0.018 & 0.187 & 0.017 \\
\hline Distal & 1.735 & 0.07 & 0.8 & 0.0 & 0.780 & & 0.190 & 0.019 \\
\hline Distance $=3$ & 1.774 & 0.078 & 0.878 & 0.009 & 0.773 & 0.032 & 0.170 & 0.01 \\
\hline No distractor & 1.733 & 0.075 & 0.876 & 0.008 & 0.876 & 0.025 & 0.172 & 0.01 \\
\hline
\end{tabular}




\section{GENERAL DISCUSSION}

The present findings show that, at the location of an irrelevant singleton, there is first an increased sensitivity, which is followed by a reduced sensitivity, relative to a condition in which there is no distractor present. The increased sensitivity implies that spatial attention was directed at the location of the distractor singleton (e.g., Handy et al., 1999; Hawkins et al., 1990) whereas the reduced sensitivity suggests that there was inhibition at the location of the distractor (e.g., Handy et al., 1999).

This pattern of results showing initial facilitation followed by inhibition is typically associated with studies investigating IOR (Posner \& Cohen, 1984). Typically, at short SOAs, target detection performance is generally better at the location where the cue is presented than at the uncued location, reflecting the benefit of exogenous shifts of attention to the cued location. However, at longer SOAs $(>250 \mathrm{msec})$, performance is worse at the cued location. Note that finding a biphasic pattern (initial facilitation, followed by inhibition of return) has usually been reported in studies in which an abrupt onset cue was used to draw attention exogenously to a location in space. The present study shows that one can obtain such a biphasic pattern not only with exogenous abrupt onset cues, but also with irrelevant static singletons.

Experiment 2 provides strong evidence that regardless of search strategies, attention is captured by the most salient singleton in the display. We created conditions similar to those in Bacon and Egeth (1994), in which the target singleton was no longer the only element with a unique shape. These conditions are assumed to induce a feature search, which should have resulted in full topdown control, eliminating the capturing effect of the color distractor singleton. The results are clear: Even though the participants had to use a feature search mode, there was an increased sensitivity at the location of the color distractor singleton, indicating capture of spatial attention to the location of the color singleton. It is clear that one does not need theoretical concepts such as feature search and singleton detection mode to explain capture of attention by salient singletons (see also Theeuwes, 2004).

The observation that an initial increased sensitivity (Experiments 1 and 2) is followed by a reduced sensitivity (Experiment 3 ) provides additional evidence that attention is captured by the salient singleton. Indeed, only when spatial attention is exogenously captured can one expect a biphasic pattern of initial facilitation, followed by IOR. According to Klein (2000), IOR is the hallmark of exogenous orienting. Typically, IOR does not follow a shift of attention that is under top-down control (Posner \& Cohen, 1984; Pratt, Kingstone, \& Khoe, 1997). In other words, finding initial facilitation followed by IOR can be the result only of a shift of exogenous attention. As has recently been shown by Pratt, Sekuler, and McAuliffe (2001), if participants use a top-down attentional control setting, such as a singleton detection mode, one will not find IOR effects (but see Gibson \& Amelio, 2000). Finding IOR in the present study adds to the notion that at- tentional capture by static singletons is not due to some top-down strategy used by the participant but is genuinely exogenous in origin (see also Theeuwes \& Godijn, 2002, for a similar argument).

The present data are in line with earlier claims of Theeuwes (1991, 1992, 1994, 1996, 2004), who argued that the preattentive detection of a salient singleton results in a compulsory shift of attention to the location of the salient singleton. Theeuwes argued that this shift is purely bottom-up or stimulus driven and cannot be modulated by top-down attention or behavioral goals. The shift of spatial attention to the location of the singleton implies that the singleton is selected for further processing. If this singleton is the target, a response is made. If it is not the target, there may be rapid disengagement of attention from that location. The present study shows that this rapid disengagement may be accomplished through inhibitory processes. According to this notion, preattentive processing is driven exclusively by bottom-up factors, such as salience (e.g., Theeuwes, 1991, 1992, 1994), with a role for top-down factors (possibly through inhibition) only later in processing (Theeuwes et al., 2000).

The present findings are consistent with data reported by Kim and Cave (1999), employing the additional singleton search task (e.g., as in Theeuwes, 1992) in combination with a probe detection task. Kim and Cave presented probes either 60 or $150 \mathrm{msec}$ after the presentation of the search display at the location of the target and the location of the distractor. It was hypothesized that if the early preattentive processing is driven solely by bottom-up salience, as has been suggested by Theeuwes (1991, 1992), the location of the salient distractor singleton should be attended first. Therefore, the probe RT at the distractor location should be shorter than that at any of the other locations in the short-SOA condition, regardless of whether the unique feature was relevant or not. On the other hand, if top-down control is possible somewhat later in time, as the present experiments suggest, it would be expected that in the late-SOA condition, attention would no longer be at the distractor location but, instead, would be at the location of the target singleton. For conditions in which the target and the distractor were locally unique (and therefore, salient enough), Kim and Cave did indeed find these results. At the $60-\mathrm{msec} \mathrm{SOA}$, the probe RT at the location of the distractor singleton was about $20 \mathrm{msec}$ shorter than that at the target singleton location. At the 150-msec SOA, however, this pattern was reversed: The probe RT at the target location was about $15 \mathrm{msec}$ shorter than that at the distractor location. The bottom line is that Kim and Cave also showed that after $150 \mathrm{msec}$, attention is no longer at the location of the distractor but, instead, is at the location of the target.

As has been noted, the biphasic pattern of an increased and decreased sensitivity at the location of the irrelevant singleton suggests the operation of IOR. Whether the observed inhibition is bottom-up, in the sense that it is a byproduct of exogenous attentional capture, or top-down, because active inhibition allows fast disengagement of attention, is an open question. Lamy and Egeth (2003) ar- 
gued that spatial inhibition needed for recovery from capture should not be labeled as IOR, since it is found with SOAs as short as $150 \mathrm{msec}$. Even though it is conceivable that it is some type of inhibition not related to IOR, the time course of inhibition may not be an adequate marker, because Danziger and Kingstone (1999) have shown that IOR can be observed very early in time (i.e., within $50 \mathrm{msec}$ ). In addition, Theeuwes et al. (2000) showed that disengagement in an attentional capture task is very fast (within $150 \mathrm{msec}$ ), and maybe this (fast) disengagement is nothing else than IOR.

\section{REFERENCES}

Bacon, W. F., \& Egeth, H. E. (1994). Overriding stimulus-driven attentional capture. Perception \& Psychophysics, 55, 485-496.

DANZIGER, S., \& Kingstone, A. (1999). Unmasking the inhibition of return phenomenon. Perception \& Psychophysics, 61, 1024-1037.

Folk, C. L., \& Remington, R. W. (1998). Selectivity in distraction by irrelevant featural singletons: Evidence for two forms of attentional capture. Journal of Experimental Psychology: Human Perception \& Performance, 24, 847-858.

Folk, C. L., \& Remington, R. W. (in press). Top-down modulation of preattentive processing: Testing the recovery account of contingent capture. Visual Cognition.

Folk, C. L., Remington, R. W., \& Johnston, J. C. (1992). Involuntary covert orienting is contingent on attentional control settings. Journal of Experimental Psychology: Human Perception \& Performance, 18, 1030-1044.

Gibson, B. S., \& Amelio, J. (2000). Inhibition of return and attentional control settings. Perception \& Psychophysics, 62, 496-504.

Handy, T. C., Jha, A. P., \& Mangun, G. R. (1999). Promoting novelty in vision: Inhibition of return modulates perceptual-level processing. Psychological Science, 10, 157-161.

Handy, T. C., KingSTone, A., \& Mangun, G. R. (1996). Spatial distribution of visual attention: Perceptual sensitivity and response latency. Perception \& Psychophysics, 58, 613-627.

Hawkins, H. L., Hillyard, S. A., Luck, S. J., Mouloua, M., DownING, C. J., \& WoodWARD, D. P. (1990). Visual attention modulates signal detectability. Journal of Experimental Psychology: Human Perception \& Performance, 16, 802-811.

ITTI, L., \& KoсH, C. (2001). Computational modeling of visual attention. Nature Reviews Neuroscience, 2, 194-203.

Kahneman, D., Treisman, A., \& Burkell, J. (1983). The costs of visual filtering. Journal of Experimental Psychology: Human Perception \& Performance, 9, 510-522.

KIM, M.-S., \& CAVE, K. R. (1999). Top-down and bottom-up attentional control: On the nature of interference from a salient distractor. Perception \& Psychophysics, 61, 1009-1023.

KLEIN, R. M. (2000). Inhibition of return. Trends in Cognitive Sciences, $4,138-147$.

Koch, C., \& Ullman, S. (1985). Shifts in selective visual attention: Towards the underlying neural circuitry. Human Neurobiology, 4, 219-227.

LAmy, D., \& Egeth, H. E. (2003). Attentional capture in singletondetection and feature-search modes. Journal of Experimental Psychology: Human Perception \& Performance, 29, 1003-1020.

Lamy, D., Tsal, Y., \& Egeth, H. E. (2003). Does a salient distractor capture attention early in processing? Psychonomic Bulletin \& Review, 10, 621-629.

Macmillan, N. A., \& Creelman, C. D. (1996). Triangles in ROC space: History and theory of "nonparametric" measures of sensitivity and response bias. Psychonomic Bulletin \& Review, 3, 164-170.
Posner, M. I., \& Cohen, Y. (1984). Components of visual orienting. In H. Bouma \& D. G. Bouwhuis (Eds.), Attention and performance X: Control of language processes (pp. 531-556). Hillsdale, NJ: Erlbaum.

Pratt, J., Kingstone, A., \& Khoe, W. (1997). Inhibition of return in location- and identity-based choice decision tasks. Perception \& Psychophysics, 59, 964-971.

Pratt, J., Sekuler, A. B., \& McAuliffe, J. (2001). The role of attentional set on attentional cueing and inhibition of return. Visual Cognition, 8, 33-46.

RuZ, M., \& LuPí́Ñ̃z, J. (2002). Attentional capture and exogenous orienting: Upon their automaticity and sensitivity to endogenous control Psicológica, 23, 283-309.

SAGI, D., \& JulEsZ, B. (1984). Detection versus discrimination of visual orientation. Perception, 13, 619-628.

Theeuwes, J. (1990). Perceptual selectivity is task dependent: Evidence from selective search. Acta Psychologica, 74, 81-99.

Theeuwes, J. (1991). Cross-dimensional perceptual selectivity. Perception \& Psychophysics, 50, 184-193.

Theeuwes, J. (1992). Perceptual selectivity for color and form. Perception \& Psychophysics, 51, 599-606.

THEeuweS, J. (1994). Stimulus-driven capture and attentional set: Selective search for color and visual abrupt onsets. Journal of Experimental Psychology: Human Perception \& Performance, 20, 799-806.

Theeuwes, J. (1996). Perceptual selectivity for color and form: On the nature of the interference effect. In A. F. Kramer, M. G. H. Coles, \& G. D. Logan (Eds.), Converging operations in the study of visual attention (pp. 297-314). Washington, DC: American Psychological Association Press.

TheEuwes, J. (2004). Top-down search strategies cannot override attentional capture. Psychonomic Bulletin \& Review, 11, 65-70.

Theeuwes, J., Atchley, P., \& Kramer, A. F. (2000). On the time course of top-down and bottom-up control of visual attention. In S. Monsell \& J. Driver (Eds.), Control of cognitive processes: Attention and performance XVIII (pp. 105-125). Cambridge, MA: MIT Press.

Theeuwes, J., \& GodiJn, R. (2001). Attention and oculomotor capture. In C. L. Folk \& B. S. Gibson (Eds.), Attraction, distraction, and action: Interdisciplinary perspectives on attentional capture (pp. 121150). Amsterdam: Elsevier.

Theeuwes, J., \& GodiJn, R. (2002). Irrelevant singletons capture attention: Evidence from inhibition of return. Perception \& Psychophysics, 64, 764-770.

Theeuwes, J., Kramer, A. F., \& Kingstone, A. (2004). Attentional capture modulates perceptual sensitivity. Psychonomic Bulletin \& Review, 11, 551-554.

Turatto, M., \& Galfano, G. (2001). Attentional capture by color without any relevant attentional set. Perception \& Psychophysics, 63 , 286-297.

Yantis, S., \& Egeth, H. E. (1999). On the distinction between visual salience and stimulus-driven attentional capture. Journal of Experimental Psychology: Human Perception \& Performance, 25, 661-676.

\section{NOTES}

1. Unlike $d^{\prime}, A^{\prime}$ is a signal detection measure that does not assume normal distributions of signal and noise (see Macmillan \& Creelman, 1996).

2. Lamy and Egeth (2003) also discussed another inhibitory process, referred to as preparatory feature-based inhibition. According to Lamy and Egeth, this type of inhibition will result in resistance to capture, as they observed in their Experiments 5 and 6.

(Manuscript received March 29, 2004; revision accepted for publication February 14, 2005.) 